\title{
Benfotiamine and Type 2 Diabetes Mellitus
}

\section{Abstract}

Benfotiamine (BFT) supplementation may provide benefits in the prevention of other diabetes-related vascular and neuronal comorbidities. The mechanism of BFT influence on diabetic angio, neuropathies pathogenesis is not well-known. Thus, further investigations aimed to understand the mechanism of action and for confirmation of the beneficial effect of BFT on biochemical parameters, dynamics of independent cardiovascular tests, daily monitoring of electrocardiography, arterial wall stiffness parameters among patients with type 2 diabetes mellitus, diabetic angio-, neuropathies and its associated comorbidities may be needed to validate this clinical findings.

Keywords: Type 2 diabetes mellitus; Cardiovascular diseases; Autonomic neuropathy; Benfotiamine

\author{
Mini Review \\ Volume 7 Issue 1 - 2018 \\ Victoria Serhiyenko*, Marta Hotsko, Oksana \\ Snitynska and Alexandr Serhiyenko \\ Department of Endocrinology, Danylo Galytsky Lviv National \\ Medical University, Ukraine \\ *Corresponding author: Victoria Serhiyenko, Department \\ of Endocrinology, Danylo Halytsky Lviv National Medical \\ University, Ukraine, 69, Pekarska str., 79010, Ukraine; Tel: \\ +38-322-76-94-96; Email: 965554@ukrnet; \\ serhiyenkoa@gmail.com
}

Received: January 21, 2018 | Published: February 15, 2018

\section{Introduction}

The number of people with type 2 diabetes mellitus (T2DM) has been increasing worldwide [1]. The majority of patients with long-term course of diabetes mellitus (DM) (mainly T2DM) are diagnosed with cardiovascular diseases. Conditionally, there are two main forms of heart disease in case of DM: diabetic cardiomyopathy and ischemic heart disease (IHD). There is a metabolic stage (actual cardiomyopathy); metabolic-ischemic stage-IHD; myocardial infarction (MI); dystrophic coronary cardiosclerosis; cardiac autonomic neuropathy (CAN) [2]. Pathogenetic treatment includes: balanced diet and physical activity; reducing insulin resistance; optimization of glycaemic control; treatment of hyperlipidaemia; correction of metabolic abnormalities in myocardium; prevention and treatment of thrombosis; antioxidants, first of all $\alpha$-lipoic acid (ALA); use of $\omega-3$ polyunsaturated fatty acids; vasodilators; fat-soluble vitamin $\mathrm{B}_{1}$ (benfotiamine); treatment of concomitant diseases and syndromes (hypertension, heart failure and arrhythmias) and others $[3,4]$. The aim of this mini-review was to analyze the latest evidence about the effects of benfotiamine (BFT) (a lipid-soluble thiamine derivative with higher bioavailability than thiamine) on some metabolic and functional parameters in T2DM patients with diabetic angio-, neuropathies.

\section{Discussion}

Diabetes might be considered as thiamine deficiency (TD) state, if not in absolute terms at least relative to the increased requirements deriving from accelerated and amplified glucose metabolism in non-insulin dependent tissues that, like the vessel wall, are prone to complications [5,6]. Some oxidized thiamine metabolites could also play role in pathogenesis of diabetes complications. It is reported that plasma thiamine levels are decreased by $75 \%$ in T2DM patients [7]. The conventional indicator of thiamine sufficiency, erythrocyte transketolase (TKT) activity, is masked in clinical DM by increased protein levels of thiamine transporter-1 (THTR-1) and THTR-2. The TD in clinical DM may increase the fragility of vascular cells to the adverse effects of hyperglycemia and there by the increase of the risk of developing microvascular complications. A suppression of TKT activity, and subsequent down-regulation of the hexose monophosphate (HMP) shunt, resulting in accumulation of glyceraldehyde 3-phosphate (GA3P), fructose 6-phosphate (F6-P), and dihydroxyacetone phosphate may be at least one mechanism in the development of DM-induced vascular damage and other comorbidities [5,8-10].

It is hypothesized that genetic variability in TKT might contribute to susceptibility to early diabetic neuropathies (DN). TKT is indeed pertinent to the pathogenesis of diabetic microangiopathic complications. When activated in the cell by thiamine, TKT determines the diversion of F6-P and GA3P to the pentose-phosphates pathway (PPP), in this way it exercises a protective effect against three pathogenetic mechanisms of microvascular complications, i.e. the glucose-driven hexosamine, protein kinase C (PKC), and advanced glycation end products (AGE's) pathways [11]. Therefore, changes in thiamine levels may be masked by an increase in THTR expression [12]. Thiamine and its derivatives have been demonstrated to prevent the activation of the biochemical pathways [increased flux through the polyol pathway, formation of AGE's, activation of PKC, and increased flux through the hexosamine biosynthesis pathway (HBP)] induced by hyperglycemia in DM. TD plays a role in the diabetic endothelial vascular diseases (micro- and macroangiopathy), lipid profile, retinopathy, nephropathy, cardiopathy, and neuropathy [12].

Thiamine acts as a coenzyme for TKT and for the pyruvate dehydrogenase and $\alpha$-ketoglutarate dehydrogenase complexes, enzymes which play a fundamental role in intracellular glucose metabolism. TKT and glucose-6-phosphate dehydrogenase, the rate-limiting enzymes of the PPP, are inhibited in the diabetic heart under basal conditions [13]. There is sufficient evidence to indicate that THTR's and TKT activity are suppressed in DM. 
Restoring TKT activity via BFT or thiamin supplementation can increase the flux of glucose into HMP shunt, and also increase flux of GA3P and F6P into HMP shunt and away from hyperglycemiainduced pathways that lead to vascular damage [9].

Experiences from cardiology indicate that long-term increases in heart rate variability and reduction in sudden cardiac death have only been shown with lipophilic agents that readily penetrate the blood nerve/blood brain barrier. In accordance with these observations experimental data indicate a preventive effect of BFT on the development of diabetic CAN [14]. Thiamine supplementation can prevent hyperglycemia-driven reductions in cell replication and proliferation as well as decreasing AGE's formation. BFT has been shown to prevent increased markers of HBP activity, intracellular AGE's formation, intracellular PKC activity and the nuclear factor kappa B (NF- $\mathrm{BB}$ ) activation seen with in vitro hyperglycemic damage [15]. High-dose therapy of BFT suppressed AGE's accumulation in the peripheral nerve and reversed DN potentially by reducing the levels of triose phosphates via activation of TKT [16]. Oral BFT in combination with the antioxidant ALA treatment normalizes production of angiopoietin-2, a marker of increased intracellular methylglyoxals in endothelial cells which contribute to AGE's formation, and $\mathrm{N}$-acetylglucose modified protein, a marker of HBP activity. Treatment with BFT has been shown to reduce activation of the polyol pathway of glucose metabolism and to increase TKT expression in the presence of hyperglycemia $[5,15]$. Activation of AGE receptors in DM, found on cardiomyocytes, pericytes, and podocytes, stimulates postreceptor signaling, intracellular reactive oxygen species formation, and altered gene expression, leading to vascular damage $[9,17]$.

BFT significantly reduced inflammatory $(10-300 \mathrm{mg} / \mathrm{kg})$ and neuropathic $(75-300 \mathrm{mg} / \mathrm{kg})$ nociception in non-diabetic and diabetic rats. BFT significantly decreased production of proinflammatory mediators such as inducible form of nitric oxide (NO) synthase and NO; cyclooxygenase-2, heat-shock protein 70, tumor necrosis factor- $\alpha$, interleukin (IL)- 6 , whereas it increased antiinflammatory IL-10 production in lipopolysaccharide-stimulated BV-2 microglia. Moreover, BFT suppressed the phosphorylation of extracellular signal-regulated kinases 1/2 (ERK1/2), c-Jun $\mathrm{N}$-terminal kinases (JNK) and a serine/threonine protein kinase (Akt/PKB). Treatment with specific inhibitors revealed that BFTmediated suppression of NO production was via JNK1/2 and Akt pathway, while the cytokine suppression includes ERK1/2, JNK1/2 and Akt pathways. Finally, the potentially protective effect is mediated by the suppression of translocation of NF- $\kappa B$ in the nucleus [18]. These findings suggest that thiamine/BFT may plays a role in modulating the inflammatory process [12]. The explanations about positive thiamine effects were confined to hydrophobic thiamine metabolites that fulfill an important function under oxidative stress (OS) and nitrosyl stress [6].

Dysautonomia is a broad term that describes any disease or malfunction of the autonomic nervous system (ANS). The various forms of mild to moderate vitamin deficiencies result in functional changes in the ANS. It is hypothesized that the predictable loss of efficiency in oxidative metabolism is the key to understanding the association of dysautonomia with many different diseases. Mild to moderate hypoxia and/or TD both give rise to exaggeration of centrally controlled mechanisms involved in all survival reflexes, mediated normally through a balanced reaction of the ANS and endocrine system. Chronic exposure to hypoxia increases the activity of the sympathetic nervous system and adrenal medulla and TD induces an early functionally significant central muscarinic cholinergic lesion [19]. BFT treatment counters DMinduced cardiac mechanical dysfunction at the cellular level, associated with reduction in OS but not AGE's formation or cardiac protein carbonyl formation. This apparent discrepancy in BFTelicited action on AGE's formation and OS (the oxidized/reduced glutathione ratio) seems to indicate that other mechanism(s) may predominantly contribute to diabetes-induced OS and cardiac contractile dysfunction in current experimental setting. Possible candidates may include alteration in glucose metabolism and PKC activation [20], although further study is warranted to verify involvement of these signaling pathways and beneficial effects of BFT against DM complications.

A decrease in the blood supply to the heart caused by atherosclerosis or thrombosis is known to induce MI [12]. The results of our study revealed that the appointment of BFT to patients with T2DM and advances stage of CAN were accompanied by a decrease of the thromboxane B2 (TxB2) concentration and TxB2/6-keto-prostaglandin $\mathrm{F} 1 \alpha$ ratio, which may contribute to the improvement of the functional state of the prostacyclin I2TxA2 system [3]. Benfotiamine supplementation for $14 \mathrm{wks}$ (100 $\mathrm{mg} / \mathrm{kg} / \mathrm{d}$ ) to streptozotocin-induced diabetic mice completely corrected hyperglycemia-induced disruptions in $\mathrm{Ca}^{2+}$ homeostasis and mechanical functioning of cardiomyocytes [9]. Cardiac OS is involved in heart failure that is induced by thiamine deprivation in rats. These findings suggest that thiamine modulates OS [12]. Endothelial NO synthase (eNOS) and NO may play an important role in attenuating cardiac remodeling and apoptosis. BFT reduces OS and activates eNOS to enhance the generation and bioavailability of NO, and it subsequently improves the integrity of vascular endothelium to prevent sodium arsenite-induced experimental vascular endothelial dysfunction [9]. CAN may contribute to an increase in artery rigidity, a cardiovascular risk factor $[11,21]$.

\section{Conclusion}

There is moderate evidence from preclinical experimental models that high-dose thiamine and BFT (1) inhibit the HMP, AGE's formation, and diacylglycerol-PKC through the TKT activation; (2) target at various surrogate markers of hyperglycemia-induced pathological processes $[8,11]$ and (3) can delay the progression of microangiopathic complications [11]. Therefore, BFT may have therapeutic potential for neurological diseases by inhibiting inflammatory mediators and enhancing anti-inflammatory factor production $[22,12,21]$. The positive influences of BFT on such metabolic and functional parameters are partly confirmed by its neurotropic, cardioprotective, angioprotective and cytoprotective properties; this findings suggests the feasibility of its usage in the complex treatment of patients with T2DM and diabetic angio-, neuropathies. 


\section{Conflict of Interest}

None.

\section{Acknowledgement}

None.

\section{References}

1. Ogurtsova K, da Rocha Fernandes JD, Huang Y, Linnenkamp U, Guariguata L, et al. (2017) IDF Diabetes Atlas: Global estimates for the prevalence of diabetes for 2015 and 2040. Diabetes Res Clin Pract 128(1): 40-50.

2. Serhiyenko VA, Serhiyenko AA (2015) Diabetic cardiac autonomic neuropathy: Do we have any treatment perspective. World J Diabetes 6(2): 245-258.

3. Vinik AI, Maser RE, Ziegler D (2010) Neuropathy: the crystal ball for cardiovascular disease? Diabetes Care 33(7): 1688-1690.

4. Tandon N, Ali MK, Narayan KM (2012) Pharmacologic prevention of microvascular and macrovascular complications in diabetes mellitus. Implications of the results of recent clinical trials in type 2 diabetes. Am J Cardiovasc Drugs 12(1): 7-12.

5. Berrone E, Beltramo E, Solimine C, Ape AU, Porta M (2006) Regulation of intracellular glucose and polyol pathway by thiamine and benfotiamine in vascular cells cultured in high glucose. J Biol Chem 281(14): 9307-9313

6. Rašić-Milutinović Z, Gluvić Z, Peruničić-Peković G, Miličević D, Lačković M, et al. (2014) Improvement of heart rate variability with benfothiamine and alpha-lipoic acid in type 2 diabetic patients Pilot study. J Cardiovasc Pharmacol Ther 2(1): 1-7.

7. Thornalley PJ, Babaei-Jadidi H, Rabbani N, Antonysunil A, Larkin J, et al. (2007) High prevalence of low plasma thiamine concentration in diabetes linked to a marker of vascular disease. Diabetologia 50(10): 164-217.

8. Pácal L, Kuricová K, Kaňková K (2014) Evidence for altered thiamine metabolism in diabetes: is there a potential to oppose gluco- and lipotoxicity by rational supplementation? World J Diabetes 5(3): 288-295.

9. Moss CJ, Mathews ST (2013) Thiamin status and supplementation in the management of diabetes mellitus and its vascular comorbidities. Vitam Min 2: 111.

10. Stirban A, Pop A, Tschoepe D (2013). A randomized, doubleblind, crossover, placebo-controlled trial of 6 weeks benfotiamine treatment on postprandial vascular function and variables of autonomic nerve function in Type 2 diabetes. Diabet Med 30(10): 1204-1208.

11. Spallone V (2017) Might genetics play a role in understanding and treating diabetic polyneuropathy? Diabetes Metab Res Rev 33(4): 2882-2886.

12. Luong KV, Nguyen LT (2012) The impact of thiamine treatment in the diabetes mellitus. J Clin Med Res 4(3): 153-160.

13. Katare RG, Caporali A, Emanueli C, Madeddu P (2010). Benfotiamine improves functional recovery of the infarcted heart via activation of pro-survival G6PD/Akt signaling pathway and modulation of neurohormonal response. J Mol Cell Cardiol 49(4): 625-638.

14. Kempler P (2003) Review: Autonomic neuropathy: a marker of cardiovascular risk. Br J Diabetes Vasc Dis 3(2): 84-90.

15. Page L, Laight D, Cummings MH (2011) Thiamine deficiency in diabetes mellitus and the impact of thiamine replacement on glucose metabolism and vascular disease. Int J Clin Pract 65(6): 684-690.

16. Stracke H, Gaus W, Achenbach U, Federlin K, Bretzel RG (2008) Benfotiamine in diabetic polyneuropathy (BENDIP): Results of a randomised, double blind, placebo-controlled clinical study. Exp Clin Endocrinol Diabetes 116(10): 600-605.

17. Sergienko VA, Segin VB, Samir A, Sergienko AA (2013) The effect of long-chain polyunsaturated higher omega-3 fatty acids, benfotiamine and alpha-lipoic acid on the lipid metabolism in patients with diabetes mellitus type 2 and cardiovascular autonomic neuropathy. Zhurnal nevrologii i psikhiatrii imeni S.S. Korsakova (Russian) 113(11): 54-58.

18. Bozic I, Savic D, Stevanovic I, Perovic S, Nedeljkovich N, et al. (2015) Benfotiamine upregulates antioxidative system in activated BV-2 microglia cells. Front Cell Neurosci 9: 351.

19. Lonsdale D (2009) Dysautonomia, a heuristic approach to a revised model for etiology of disease. J Evid Based Complementary Altern Med 6(1): 3-10.

20. Brownlee M (2001) Biochemistry and molecular cell biology of diabetic complications. Nature 414(6865): 813-820.

21. Serhiyenko VA, Serhiyenko AA (2018) Cardiac autonomic neuropathy: Risk factors, diagnosis and treatment. World J Diabetes 9(1): 1-24.

22. Shoeb M, Ramana KV (2012) Anti-inflammatory effects of benfotiamine are mediated through the regulation of the arachidonic acid pathway in macrophages. Free Radic Biol Med 52(1): 182-190. 